\title{
Synthesis and Antimicrobial Activities of Some New Synthesized Imide and Schiff's Base Derivatives
}

\author{
Nermien M. Sabry, ${ }^{1,2}$ Eman M. Flefel, ${ }^{3}$ Mohamed A. Al-Omar, ${ }^{4}$ and Abd El-Galil E. Amr ${ }^{2,5,5}$ \\ ${ }^{1}$ Chemistry Department, Girls College of Science, King Khalid University, Abha 9004, Saudi Arabia \\ ${ }^{2}$ Applied Organic Chemistry Department, National Research Center, Dokki, Cairo 12622, Egypt \\ ${ }^{3}$ Photochemistry Department, National Research Center, Dokki, Cairo 12622, Egypt \\ ${ }^{4}$ Pharmaceutical Chemistry Department, College of Pharmacy, King Saud University, Riyadh 11451, Saudi Arabia \\ ${ }^{5}$ Drug Exploration \& Development Chair, College of Pharmacy, King Saud University, Riyadh 11451, Saudi Arabia
}

Correspondence should be addressed to Nermien M. Sabry; nermien_m_sabry@yahoo.com

Received 18 January 2012; Accepted 16 April 2012

Academic Editor: Patricia E. Allegretti

Copyright (C) 2013 Nermien M. Sabry et al. This is an open access article distributed under the Creative Commons Attribution License, which permits unrestricted use, distribution, and reproduction in any medium, provided the original work is properly cited.

A series of 2,6-bis(substituted thiazolopyrimi-dinyl) pyridine (2a,b) and corresponding Schiff's bases (3a-j) were synthesized from 2,6-bis-(3-amino-2-methyl-4-oxo-9-substituted-3,4-dihydropyrido-[30,20:4,5]-thieno[3,2-d]pyrimidin-7-yl)pyridines (1a,b) as starting materials. The compounds 1a,b were reacted with 2,3,4,5-tetrachlorophthalic anhydride in glacial acetic acid to give the corresponding bis-imides (2a,b). But they are treated with aromatic aldehydes in refluxing ethanol to afford the Schiff's base derivatives $(\mathbf{3 a} \mathbf{a} \mathbf{j})$. The antimicrobial screening showed that many of these newly synthesized compounds had good antimicrobial activities comparable to streptomycin and fusidic acid as positive controls. The detailed synthesis, spectroscopic data, and antimicrobial activities of the synthesized compounds were reported.

\section{Introduction}

Antimicrobial agents reduce or completely block the growth and multiplication of bacteria and are helpful in the treatment of various infectious diseases like meningitis, malaria, tuberculosis, pneumonia, AIDS, and so forth. The compounds with the structure of $-\mathrm{C}=\mathrm{N}$ - (azomethine group) are known as Schiff bases, which are usually synthesized from the condensation of primary amines and active carbonyl groups. Schiff bases derived from aromatic amines and aromatic aldehydes have a wide variety of applications in many fields, for example, biological, inorganic, and analytical chemistry [1-4]. In addition, Schiff bases and heterocyclic ring are important class of compounds in medicinal and pharmaceutical field [5-8]. Recently, in our previous work, Schiff bases show biological properties including antibacterial, antifungal antitumor, analgesic, and anti-inflammatory activities [9-18]. In view of these observations and in continuation of our previous work in heterocyclic chemistry, we synthesized some new heterocyclic imide and Schiff base derivatives containing pyridine, thiophene, and pyrimidine rings for the evaluation of antimicrobial activity compared to streptomycin and fusidic acid as positive controls.

\section{Experimental}

2.1. Chemistry. Melting points were determined on open glass capillaries using an Electrothermal IA 9000 SERIES digital melting point apparatus and are uncorrected. Analytical data were obtained from the Microanalytical Unit, Cairo University, Egypt. IR (KBr) spectra were recorded on a FT IR$8201 \mathrm{PC}$ spectrophotometer. ${ }^{1} \mathrm{H}-\mathrm{NMR}$ and ${ }^{13} \mathrm{C}-\mathrm{NMR}$ spectra were determined on Jeol FTGNM-EX 270, $270 \mathrm{MHz}$ with DMSO- $\mathrm{d}_{6}$ as the solvent. Mass spectra were recorded on a Finnigan SSQ 7000 spectrometer using EI and run at $70 \mathrm{eV}$. 
2.2. Synthesis of 2,6-Bis(2-methyl-4-oxo-3-(tetrachloro-phthalimido)-9-Aryl-3,4-dihydropyrido-[30,20:4,5] thieno-[3,2d]pyrimidin-7-yl)pyridines (2a,b)

2.2.1. General Procedure. A mixture of $\mathbf{1 a}, \mathbf{b}(1 \mathrm{mmol})$ and 2,3,4,5-tetrachlorophthalic anhydride $(0.56 \mathrm{~g}, 2 \mathrm{mmol})$ was refluxed in glacial acetic acid $(30 \mathrm{~mL})$ for $6 \mathrm{~h}$. The reaction mixture was cooled, and the obtained product was collected by filtration, dried, and crystallized from $\mathrm{DMF} / \mathrm{H}_{2} \mathrm{O}$ to give the title products $\mathbf{2} \mathbf{a}, \mathbf{b}$, respectively.

2a. Yield (66\%), m.p. $>300^{\circ} \mathrm{C}$. IR (KBr): 3135, 1682, $1600 \mathrm{~cm}^{-1} ;{ }^{1} \mathrm{H}$ NMR $\delta: 2.35\left(\mathrm{~s}, 6 \mathrm{H}, 2 \mathrm{CH}_{3}\right), 6.80-7.60(\mathrm{~m}$, $10 \mathrm{H}, \mathrm{Ar}-\mathrm{H}), 8.00-8.20$ (m, 3H, pyrid-H), 8.40 (s, 2H, 2C-5' $\mathrm{H}) ;{ }^{13} \mathrm{C}$ NMR $\delta: 19.55,120.85,122.02,126.58,127.25,128.65$, $129.08,129.12,133.24,137.75,137.85,137.95,138.05,145.15$, $149.45,154.65,155.35,155.66,164.76,165.18,166.74$; MS (EI): $m / z 1227 ; \mathrm{C}_{53} \mathrm{H}_{21} \mathrm{Cl}_{8} \mathrm{~N}_{9} \mathrm{O}_{6} \mathrm{~S}_{2}$ (1227.54): calcd. C 51.86\%, $\mathrm{H} 1.72 \%, \mathrm{Cl} 23.10 \%, \mathrm{~N} 10.27 \%$, S 5.22\%; found C $51.79 \%, \mathrm{H}$ $1.66 \%, \mathrm{Cl} 23.04 \%$, N 10.22\%, S 5.16\%.

2b. Yield (56\%), m.p. $>300^{\circ} \mathrm{C}$. IR (KBr): 3125, 1679, $1604 \mathrm{~cm}^{-1} ;{ }^{1} \mathrm{H}$ NMR $\delta: 2.20\left(\mathrm{~s}, 6 \mathrm{H}, 2 \mathrm{CH}_{3}\right), 7.25-7.75(\mathrm{~m}$, $6 \mathrm{H}$, thiophene-H), 8.05-8.25 (m, 3H, pyrid- $\mathrm{H}), 8.45(\mathrm{~s}, 2 \mathrm{H}$, $\left.2 \mathrm{C}-5^{\prime}-\mathrm{H}\right) ;{ }^{13} \mathrm{C}$ NMR $\delta: 19.56,121.05,122.15,125.05,126.60$, $126.98,127.18,128.60,133.18,137.80,137.88,138.02$, 138.15, 145.25, 145.68, 154.48, 155.38, 155.42, 164.72, 165.10, 166.68; MS (EI): $m / z$ 1239; $\mathrm{C}_{49} \mathrm{H}_{17} \mathrm{Cl}_{8} \mathrm{~N}_{9} \mathrm{O}_{6} \mathrm{~S}_{4}$ (1239.60): calcd. C $47.48 \%, \mathrm{H} 1.38 \%, \mathrm{Cl} 22.88 \%$, N 10.17\%, S 10.35\%; found C $47.42 \%, \mathrm{H} 1.33 \%$, Cl 22.84\%, N 10.12\%, S 10.30\%.

\subsection{Synthesis of 2,6-Bis(2-methyl-4-oxo-3-(substituted phenyl methylidene)amino)-9-substituted-3,4-dihydropyrido-[30, 20:4,5] thieno [3,2-d]pyrimidin-7-yl)pyridines (3a-j)}

2.3.1. General Procedure. A mixture of $\mathbf{1 a}, \mathbf{b}(1 \mathrm{mmol})$ and aromatic aldehydes, namely, benzaldehyde, 4-fluoro-, 4chloro-, 4-methyl-, or 4-methoxy-, benzaldehyde ( $2 \mathrm{mmol})$ in absolute ethanol $(25 \mathrm{~mL})$ with a few drops piperidine was refluxed for $6 \mathrm{~h}$. The reaction mixture was concentrated under reduced pressure, poured onto water, and the obtained solid was filtered off, washed with water, dried, and crystallized from the proper solvent to give the corresponding Schiff bases $(\mathbf{3} \mathbf{a}-\mathbf{j})$, respectively.

3a. Yield (62\%), m.p. $265-267^{\circ} \mathrm{C}\left(\mathrm{DMF} / \mathrm{H}_{2} \mathrm{O}\right)$. IR ( $\left.\mathrm{KBr}\right)$ : $3105,1680,1605 \mathrm{~cm}^{-1} ;{ }^{1} \mathrm{H} \mathrm{NMR} \delta: 2.18\left(\mathrm{~s}, 6 \mathrm{H}, 2 \mathrm{CH}_{3}\right)$, 7.12-7.62 (m, 20H, $4 \mathrm{Ph}-\mathrm{H}), 7.98-8.15$ (m, 3H, pyrid$\mathrm{H}), 8.42\left(\mathrm{~s}, 2 \mathrm{C}-5^{\prime}-\mathrm{H}\right), 8.76(\mathrm{~s}, 2 \mathrm{H}, 2 \mathrm{CH}=\mathrm{N}) ;{ }^{13} \mathrm{C}$ NMR $\delta$ : $19.98,121.08,121.88,126.44,127.22,128.65,129.01,129.14$, $129.15,130.75,133.78,137.76,137.82,137.95,142.85,145.25$, $149.40,154.62,155.42,155.72,165.26,166.70$; MS (EI): $m / z$ 868; $\mathrm{C}_{51} \mathrm{H}_{33} \mathrm{~N}_{9} \mathrm{O}_{2} \mathrm{~S}_{2}$ (867.99): calcd. C 70.57\%, H 3.83\%, N $14.52 \%$, S 7.39\%; found C 70.52\%, H 3.78\%, N $14.47 \%, \mathrm{~S}$ $7.33 \%$.

3b. Yield (72\%), m.p. $>300^{\circ} \mathrm{C}\left(\mathrm{DMF} / \mathrm{H}_{2} \mathrm{O}\right)$. IR $(\mathrm{KBr})$ : $3110,1682,1608 \mathrm{~cm}^{-1}$; ${ }^{1} \mathrm{H}$ NMR $\delta: 1.98\left(\mathrm{~s}, 6 \mathrm{H}, 2 \mathrm{CH}_{3}\right), 7.12-$ $7.65(\mathrm{~m}, 18 \mathrm{H}, 2 \mathrm{Ph}-\mathrm{H}), 8.05-8.23$ (m, 3H, pyrid-H), 8.56 (s, 2C-5'-H), $8.75(\mathrm{~s}, 2 \mathrm{H}, 2 \mathrm{CH}=\mathrm{N}) ;{ }^{13} \mathrm{C}$ NMR $\delta: 20.18$, $121.14,121.92$, 126.36, 127.28, 129.01, 129.12, 129.24, 130.45,
$131.85,136.24,137.78,137.84,137.96,142.76,145.15,149.35$, 154.60, 155.48, 155.75, 165.12, 166.68; MS (EI): $\mathrm{m} / z$ 937; $\mathrm{C}_{51} \mathrm{H}_{31} \mathrm{Cl}_{2} \mathrm{~N}_{9} \mathrm{O}_{2} \mathrm{~S}_{2}$ (936.88): C 65.38\%, H 3.34\%, Cl 7.57\%, $\mathrm{N} 13.46 \%, \mathrm{~S} 6.85 \%$; found C $65.32 \%, \mathrm{H} 3.30 \%, \mathrm{Cl} 7.52 \%, \mathrm{~N}$ $13.40 \%$, S $6.80 \%$.

3c. Yield (70\%), m.p. $214-216^{\circ} \mathrm{C}$ (EtOH/ether). IR (KBr): $3108,1678,1615 \mathrm{~cm}^{-1} ;{ }^{1} \mathrm{H}$ NMR $\delta: 1.98\left(\mathrm{~s}, 6 \mathrm{H}, 2 \mathrm{CH}_{3}\right)$, 7.06-7.66 (m, 18H, 2 Ph-H), 8.10-8.18 (m, 3H, pyrid-H), $8.62\left(\mathrm{~s}, 2 \mathrm{C}-5^{\prime}-\mathrm{H}\right), 8.78(\mathrm{~s}, 2 \mathrm{H}, 2 \mathrm{CH}=\mathrm{N}) ;{ }^{13} \mathrm{C}$ NMR $\delta: 20.06$, $115.66,121.16,121.96,126.40,127.32,129.12$, 129.14, 129.21, $130.25,137.80,137.84,137.95,142.84,145.22,149.38,154.61$, 155.52, 155.68, 164.88, 165.16, 166.60; MS (EI): $\mathrm{m} / z$ 904; $\mathrm{C}_{51} \mathrm{H}_{31} \mathrm{~F}_{2} \mathrm{~N}_{9} \mathrm{O}_{2} \mathrm{~S}_{2}$ (903.98): calcd. C 67.76\%, H 3.46\%, N $13.95 \%$, S 7.09\%; found C $67.70 \%, \mathrm{H} 3.40 \%, \mathrm{~N} 13.89 \%$, S $7.02 \%$.

3d. Yield (65\%), m.p. $273-275^{\circ} \mathrm{C}\left(\mathrm{AcOH} / \mathrm{H}_{2} \mathrm{O}\right)$. IR (KBr): 3112, 1680, $1612 \mathrm{~cm}^{-1}$; ${ }^{1} \mathrm{H}$ NMR $\delta: 1.82,2.25(2 \mathrm{~s}, 12 \mathrm{H}$, $4 \mathrm{CH}_{3}$ ), 7.12-7.58 (m, 18H, $\left.2 \mathrm{Ph}-\mathrm{H}\right), 7.98-8.22$ (m, 3H, pyrid$\mathrm{H}), 8.68\left(\mathrm{~s}, 2 \mathrm{C}-5^{\prime}-\mathrm{H}\right), 8.75(\mathrm{~s}, 2 \mathrm{H}, 2 \mathrm{CH}=\mathrm{N}) ;{ }^{13} \mathrm{C}$ NMR $\delta$ : $19.86,23.98,121.09,127.54,128.68,121.94,126.55,129.00$, $129.45,129.65,130.56,137.78,137.86,137.98,140.62,142.64$, $145.32,149.44,154.62,155.54,155.84,165.24,167.05 ; \mathrm{MS}$ (EI): $m / z$ 896; $\mathrm{C}_{53} \mathrm{H}_{37} \mathrm{~N}_{9} \mathrm{O}_{2} \mathrm{~S}_{2}$ (896.05): C 71.04\%, $\mathrm{H} 4.16 \%$, $\mathrm{N} 14.07 \%$, S 7.16\%; found C $71.00 \%, \mathrm{H} 4.11 \%, \mathrm{~N} 14.00 \%, \mathrm{~S}$ $7.12 \%$.

3e. Yield (65\%), m.p. $286-288^{\circ} \mathrm{C}\left(\mathrm{MeOH} / \mathrm{H}_{2} \mathrm{O}\right)$. IR (KBr): $3118,1679,1610 \mathrm{~cm}^{-1} ;{ }^{1} \mathrm{H}$ NMR $\delta: 1.88,3.72(2 \mathrm{~s}, 12 \mathrm{H}$, $\left.4 \mathrm{CH}_{3}\right), 6.85-7.62(\mathrm{~m}, 18 \mathrm{H}, 2 \mathrm{Ph}-\mathrm{H}), 8.01-8.24$ (m, 3H, pyrid$\mathrm{H}), 8.65\left(\mathrm{~s}, 2 \mathrm{C}-5^{\prime}-\mathrm{H}\right), 8.76(\mathrm{~s}, 2 \mathrm{H}, 2 \mathrm{CH}=\mathrm{N}) ;{ }^{13} \mathrm{C}$ NMR $\delta$ : 20.04, 55.84, 114.12, 121.16, 121.86, 125.78, 126.42, 127.24, $129.12,129.45,129.88,137.68,137.84,137.95,142.82,145.32$, $149.38,154.56,155.50,155.78,162.98,165.26,166.74 ; \mathrm{MS}$ (EI): $m / z$ 928; $\mathrm{C}_{53} \mathrm{H}_{37} \mathrm{~N}_{9} \mathrm{O}_{4} \mathrm{~S}_{2}$ (928.05): $\mathrm{C} 68.59 \%, \mathrm{H} 4.02 \%$, N 13.58\%, S 6.91\%; found C 68.53\%, H 3.98\%, N 13.52\%, S $6.86 \%$.

3f. Yield (72\%), m.p. $290-292^{\circ} \mathrm{C}\left(\mathrm{DMF} / \mathrm{H}_{2} \mathrm{O}\right)$. IR (KBr): $3118,1682,1608 \mathrm{~cm}^{-1}$; ${ }^{1} \mathrm{H}$ NMR $\delta: 1.88\left(\mathrm{~s}, 6 \mathrm{H}, 2 \mathrm{CH}_{3}\right)$, 7.14-7.65 (m, 16H, Ar-H), 8.02-8.24 (m, 3H, pyrid-H), 8.56 (s, 2C-5'-H), $8.72(\mathrm{~s}, 2 \mathrm{H}, 2 \mathrm{CH}=\mathrm{N}) ;{ }^{13} \mathrm{C}$ NMR $\delta: 20.10$, $121.32,121.95,125.15,126.65,127.23,128.02,128.60,129.12$, $131.10,133.82,137.84,137.98,138.04,142.83,145.14,149.35$, $154.68,155.44,155.76,165.18,166.66$; MS (EI): $\mathrm{m} / z$ 880; $\mathrm{C}_{47} \mathrm{H}_{29} \mathrm{~N}_{9} \mathrm{O}_{2} \mathrm{~S}_{4}$ (880.05): C 64.14\%, H 3.32\%, N 14.32\%, S $14.57 \%$; found C $64.10 \%, \mathrm{H} 3.28 \%$, N $14.27 \%$, S $14.52 \%$.

3g. Yield (68\%), m.p. 265-267 $\mathrm{C}(\mathrm{AcOH})$. IR (KBr): 3116, $1685,1614 \mathrm{~cm}^{-1} ;{ }^{1} \mathrm{H}$ NMR $\delta: 1.92\left(\mathrm{~s}, 6 \mathrm{H}, 2 \mathrm{CH}_{3}\right), 7.23-7.72$ (m, 14H, Ar-H), 8.10-8.26 (m, 3H, pyrid-H), 8.62 (s, 2C-5' $\mathrm{H}), 8.76(\mathrm{~s}, 2 \mathrm{H}, 2 \mathrm{CH}=\mathrm{N}) ;{ }^{13} \mathrm{C}$ NMR $\delta: 20.00,121.24,121.94$, $124.98,126.42,127.33,128.12,128.95,130.50,131.86,136.32$, $137.82,137.90,138.30,142.68,145.18,149.38,154.64,155.52$, 155.70, 165.16, 166.76; MS (EI): $m / z$ 949; $\mathrm{C}_{47} \mathrm{H}_{27} \mathrm{Cl}_{2} \mathrm{~N}_{9} \mathrm{O}_{2} \mathrm{~S}_{4}$ (948.94): C 59.49\%, H 2.87\%, Cl 7.47\%, N 13.28\%, S 13.52\%; found C 59.43\%, H 2.82\%, Cl 7.43\%, N 13.24\%, S 13.48\%.

3h. Yield (70\%), m.p. $266-268^{\circ} \mathrm{C}\left(\mathrm{DMF} / \mathrm{H}_{2} \mathrm{O}\right)$. IR (KBr): $31109,1682,1615 \mathrm{~cm}^{-1}$; ${ }^{1} \mathrm{H}$ NMR $\delta: 1.96\left(\mathrm{~s}, 6 \mathrm{H}, 2 \mathrm{CH}_{3}\right)$, 6.97-7.65 (m, 14H, Ar-H), 8.08-8.32 (m, 3H, pyrid-H), 8.65 $\left(\mathrm{s}, 2 \mathrm{C}-5^{\prime}-\mathrm{H}\right), 8.72(\mathrm{~s}, 2 \mathrm{H}, 2 \mathrm{CH}=\mathrm{N}) ;{ }^{13} \mathrm{C} \mathrm{NMR} \delta: 20.06$, 
TABLE 1: Antimicrobial activity of the newly synthesized compounds.

\begin{tabular}{|c|c|c|c|c|c|c|c|c|c|}
\hline \multirow{4}{*}{$\begin{array}{l}\text { Compound } \\
\text { number }\end{array}$} & \multicolumn{9}{|c|}{ Mean of inhibition zone (mm) } \\
\hline & \multicolumn{5}{|c|}{ Fungi } & \multicolumn{4}{|c|}{ Bacteria } \\
\hline & \multirow[b]{2}{*}{ R. $i$. } & \multirow[b]{2}{*}{ C. $a$. } & \multirow[b]{2}{*}{ Pen. sp } & \multirow[b]{2}{*}{ A. $n$. } & \multirow[b]{2}{*}{ Str. sp } & \multicolumn{2}{|c|}{ Gram -ve } & \multicolumn{2}{|c|}{ Gram +ve } \\
\hline & & & & & & P. s. & E.c. & S. $l$. & B. $s$. \\
\hline $2 a$ & 9 & 10 & 9 & 8 & 12 & 11 & 14 & 13 & 14 \\
\hline $2 \mathbf{b}$ & 10 & 13 & 10 & 11 & 21 & 20 & 21 & 23 & 23 \\
\hline $3 \mathbf{a}$ & 11 & 12 & 13 & 11 & 14 & 13 & 11 & 12 & 12 \\
\hline $3 \mathbf{b}$ & 12 & 12 & 10 & 11 & 10 & 7 & 9 & 9 & 11 \\
\hline $3 c$ & 17 & 16 & 18 & 17 & 22 & 23 & 24 & 23 & 21 \\
\hline $3 d$ & 13 & 12 & 12 & 13 & 11 & 13 & 12 & 10 & 9 \\
\hline $3 e$ & 12 & 12 & 12 & 11 & 13 & 9 & 14 & 14 & 13 \\
\hline $3 f$ & 12 & 10 & 11 & 11 & 20 & 21 & 20 & 21 & 19 \\
\hline $3 g$ & 13 & 14 & 16 & 16 & 11 & 11 & 12 & 12 & 13 \\
\hline $3 h$ & 22 & 21 & 21 & 20 & 12 & 24 & 22 & 23 & 23 \\
\hline $3 \mathbf{i}$ & 10 & 12 & 11 & 11 & 13 & 11 & 10 & 12 & 11 \\
\hline $3 \mathbf{j}$ & 19 & 20 & 19 & 19 & 11 & 13 & 12 & 10 & 9 \\
\hline Streptomycin & - & - & - & - & 21 & 22 & 21 & 22 & 21 \\
\hline Fusidic acid & 17 & 17 & 18 & 18 & - & - & - & - & - \\
\hline
\end{tabular}

R. i.: Rhodotorula ingeniosa, C. a.: Candida albicans, Pen. sp: Penicillium sp, A. n.: Aspergillus Niger, Str. sp: Streptomyces sp., P. sp: Pseudomonas sp, E. c.: Escherichia coli, S. l.: Streptococcus lactis, B. s.: Bacillus subtilis.

TABLE 2: Effect of different concentrations of compound $\mathbf{3 f}$ showed moderate activity on bacterial growth.

\begin{tabular}{|c|c|c|c|c|c|c|}
\hline \multirow{4}{*}{$\begin{array}{l}\text { Compound } \\
\text { number }\end{array}$} & \multirow{4}{*}{ Concentration $(\mu \mathrm{g})$} & \multicolumn{5}{|c|}{ Inhibition zone (mm) } \\
\hline & & \multirow{3}{*}{ Strep. $s p$} & \multirow{2}{*}{\multicolumn{4}{|c|}{ Gram +ve }} \\
\hline & & & & & & \\
\hline & & & B. $s$ & S. $l$ & E. $c$ & P.s. \\
\hline \multirow{4}{*}{$3 f$} & $1 \mathrm{x}$ & 20 & 21 & 20 & 21 & 19 \\
\hline & $2 \mathrm{x}$ & 24 & 22 & 22 & 23 & 22 \\
\hline & $3 x$ & 26 & 24 & 25 & 24 & 25 \\
\hline & $4 \mathrm{x}$ & 26 & 26 & 27 & 26 & 26 \\
\hline
\end{tabular}

Where $\mathrm{x}=10 \mu \mathrm{g}$.

$115.55,121.35,121.90,125.25,126.36,127.28,127.97,129.22$, $130.34,137.82,137.92,137.98,142.88,145.32,149.44,154.65$, $155.56,155.74,164.79,165.25,166.58 ; \mathrm{MS}(\mathrm{EI}): \mathrm{m} / z$ 916; $\mathrm{C}_{47} \mathrm{H}_{27} \mathrm{~F}_{2} \mathrm{~N}_{9} \mathrm{O}_{2} \mathrm{~S}_{4}$ (916.03): C 61.62\%, H 2.97\%, N 13.76\%, S $14.00 \%$; found C $61.56 \%$, H 2.92\%, N $13.70 \%$, S $13.96 \%$.

3i. Yield (58\%), m.p. $248-250^{\circ} \mathrm{C}$ (dioxane). IR (KBr): $31105,1680,1613 \mathrm{~cm}^{-1}$; ${ }^{1} \mathrm{H}$ NMR $\delta: 1.96,2.35(2 \mathrm{~s}, 12 \mathrm{H}$, $\left.4 \mathrm{CH}_{3}\right), 7.04-7.55(\mathrm{~m}, 14 \mathrm{H}, \mathrm{Ar}-\mathrm{H}), 7.99-8.18$ (m, 3H, pyrid$\mathrm{H}), 8.68\left(\mathrm{~s}, 2 \mathrm{C}-5^{\prime}-\mathrm{H}\right), 8.75(\mathrm{~s}, 2 \mathrm{H}, 2 \mathrm{CH}=\mathrm{N}) ;{ }^{13} \mathrm{C}$ NMR $\delta$ : $19.77,24.12,121.05,122.12,124.98,126.64,127.44,128.14$, $128.74,129.08,130.50,137.86,137.92,138.10,141.01,142.75$, $145.44,149.32,154.74,155.65,165.33,167.12,155.90 ; \mathrm{MS}$ (EI): $m / z$ 908; $\mathrm{C}_{49} \mathrm{H}_{33} \mathrm{~N}_{9} \mathrm{O}_{2} \mathrm{~S}_{4}$ (908.11): calcd. C 64.81\%, H $3.66 \%$, N 13.88\%, S 14.12\%; found C $64.75 \%, \mathrm{H} 3.60 \%, \mathrm{~N}$ $13.82 \%$, S $14.07 \%$.

3j. Yield (62\%), m.p. $276-278^{\circ} \mathrm{C}\left(\mathrm{DMF} / \mathrm{H}_{2} \mathrm{O}\right)$. IR (KBr): $3120,1683,1610 \mathrm{~cm}^{-1} ;{ }^{1} \mathrm{H}$ NMR $\delta: 1.88,3.68(2 \mathrm{~s}, 12 \mathrm{H}$, $\left.4 \mathrm{CH}_{3}\right), 7.10-7.68(\mathrm{~m}, 14 \mathrm{H}, \mathrm{Ar}-\mathrm{H}), 8.02-8.26(\mathrm{~m}, 3 \mathrm{H}$, pyrid-H), 8.65 (s, $\left.2 \mathrm{C}-5^{\prime}-\mathrm{H}\right), 8.70(\mathrm{~s}, 2 \mathrm{H}, 2 \mathrm{CH}=\mathrm{N}) ;{ }^{13} \mathrm{C} \mathrm{NMR}$ $\delta: 19.99,54.98,114.24,121.06,121.82,125.05,125.80,126.36$, $127.03,128.11,129.85,137.80,137.90,138.13,142.83,145.30$, $149.44,154.62,155.52,155.84,162.92,165.21,166.76$; MS (EI): $m / z$ 940; $\mathrm{C}_{49} \mathrm{H}_{33} \mathrm{~N}_{9} \mathrm{O}_{4} \mathrm{~S}_{4}$ (940.10): calcd. C 62.60\%, H $3.54 \%, \mathrm{~N} 13.41 \%$, S 13.64\%; found C $62.55 \%, \mathrm{H} 3.50 \%, \mathrm{~N}$ $13.36 \%$, S $13.60 \%$.

\subsection{Antimicrobial Screening}

2.4.1. Media. The following media were used.

(1) PDA medium: this medium was used for fungi cultivation. It consists of $4 \mathrm{~g}$ dextrose/L potatoes extract.

(2) Czapek Dox medium: it consists of $10 \mathrm{~g}$ glucose, $2 \mathrm{~g}$ $\mathrm{KNO}_{3}, 1 \mathrm{~g} \mathrm{~K} \mathrm{HPO}_{4}, 0.5 \mathrm{~g} \mathrm{KCl}, 0.5 \mathrm{~g} \mathrm{MgSO}_{4}$, and $0.05 \mathrm{~g}$ ferrous sulphate/L distilled water. This medium is specialized for bacteria cultivation.

(3) Medium 3: it consists of 10 glucose, $5 \mathrm{~g}$ peptone, 3 yeast extract, and 3 Malt extract. It is used for yeast cultivation. 


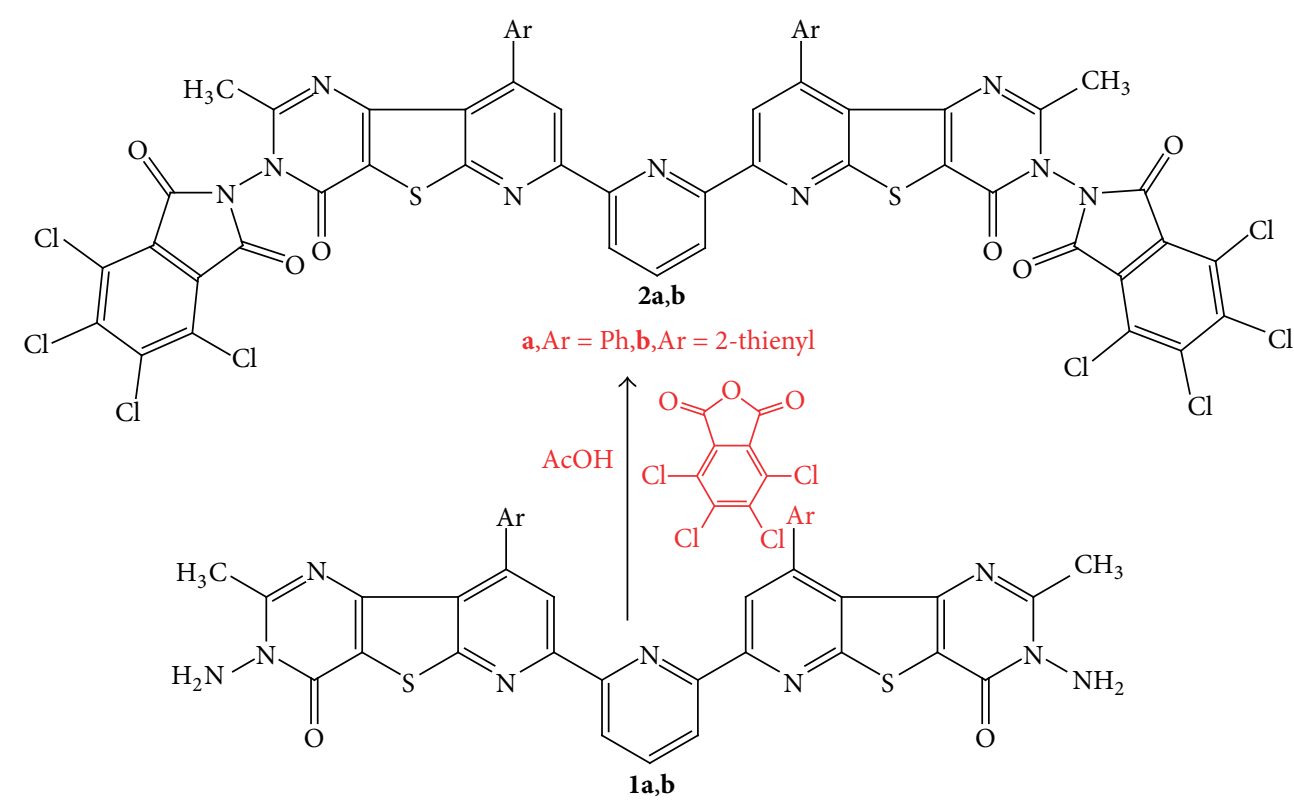

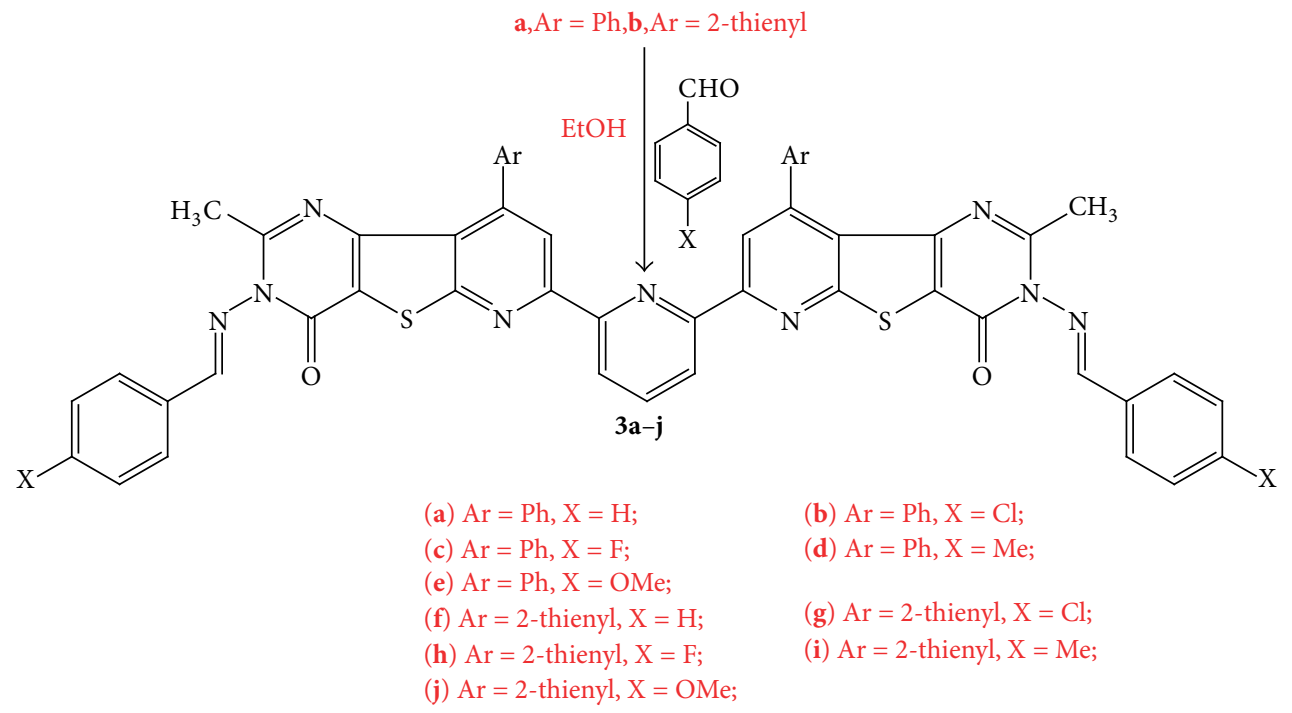

SCHEME 1: Synthetic routes of imides and Schiff bases.

\section{Results and Discussion}

3.1. Chemistry. The synthetic route is given in Scheme 1.2,6Bis(3-amino-2-methyl-4-oxo-9-substituted-3,4-dihydropyrido $\quad[30,20: 4,5]$ thieno $\quad[3,2-\mathrm{d}]$-pyrimidin-7-yl)pyridine (1a,b) was synthesized as starting material according to the literature procedure [19]. The reaction of $\mathrm{N}$-amino pyrimidine derivatives $\mathbf{1 a , b}$ with 2,3,4,5-tetrachlorophthalic anhydride in glacial acetic acid afforded the corresponding bis-imide derivatives $\mathbf{2 a}, \mathbf{b}$, respectively. Condensation of $\mathbf{1 a}, \mathbf{b}$ with aromatic aldehydes, namely, benzaldehyde, 4-fluoro-, 4-chloro-, 4-methyl-, or 4-methoxybenzaldehyde in refluxing ethanol containing a few drops of piperidine yielded the corresponding Schiff's bases $\mathbf{3} \mathbf{a}-\mathbf{j}$, respectively. All the synthesized compounds were characterized by elemental analysis, IR, ${ }^{1} \mathrm{H}$ NMR, ${ }^{13} \mathrm{C} \mathrm{NMR}$, and mass spectrometer techniques. The antimicrobial activities of the compounds $\mathbf{2 a}, \mathbf{b}$ and $\mathbf{3 a - j}$ were evaluated against three bacterial and four fungal strains.

3.2. Antimicrobial Activity. The antimicrobial activities of some synthesized compounds were determined by agar diffusion method as recommended by National Committee for Clinical Laboratory Standards (NCCLS) [20]. The compounds were evaluated for antimicrobial activity against bacteria namely, Streptomyces sp., Bacillus subtilis, Streptococcus lactis, Escherichia coli, and Pseudomonas sp and antifungal activity against various fungi (Aspergillus niger and Penicillium sp) and yeast (Candida albicans and Rhodotorula 
TABLE 3: Effect of different concentrations of compounds $\mathbf{3 c}$ and $\mathbf{3 g}$ showed moderate activity on fungal growth.

\begin{tabular}{lccccc}
\hline $\begin{array}{l}\text { Compound } \\
\text { number }\end{array}$ & $\begin{array}{c}\text { Concentration } \\
(\mu \mathrm{g})\end{array}$ & \multicolumn{5}{c}{ Inhibition zone $(\mathrm{mm})$} \\
\hline \multirow{3}{*}{$3 \mathrm{c}$} & $1 \mathrm{x}$ & 17 & 16 & 18 & 17 \\
& $2 \mathrm{x}$ & 17 & 18 & 19 & 19 \\
& $3 \mathrm{x}$ & 19 & 20 & 20 & 21 \\
& $4 \mathrm{x}$ & 20 & 22 & 20 & 21 \\
\hline $3 \mathrm{~g}$ & $1 \mathrm{x}$ & 13 & 14 & 16 & 16 \\
& $2 \mathrm{x}$ & 15 & 15 & 18 & 17 \\
& $3 \mathrm{x}$ & 18 & 17 & 20 & 20 \\
& $4 \mathrm{x}$ & 20 & 19 & 20 & 21 \\
\hline
\end{tabular}

Where $\mathrm{x}=10 \mu \mathrm{g}$.

ingeniosa). The concentrations of the tested compounds $(10 \mu \mathrm{g} / \mathrm{mL})$ were used according to modified Kirby-Bauer's disk diffusion method. The sterile discs were impregnated with $10 \mu \mathrm{g} /$ disc of the tested compound. Each tested compound was performed in triplicate. The solvent DMSO was used as a negative control, and streptomycin/fusidic acid was used as stander calculated average diameters (for triplicates) of the zone of inhibition (in $\mathrm{mm}$ ) for tested samples with that produced by the stander drugs. Four of the synthesized compounds $\mathbf{2} \mathbf{b}, \mathbf{3} \mathbf{c}, \mathbf{3} \mathbf{h}$, and $\mathbf{3} \mathbf{j}$ exhibited potent antibacterial and antifungal bioactivity compared to the stander drugs. The other tested compounds were found to exhibit a moderate of low antibacterial activity (Table 1). On the other hand, when different concentrations of the compound that exhibited a moderate antibacterial activity $\mathbf{3 f}$ were used, this compound exhibited very good antibacterial activity at higher concentrations ( $3 \mathrm{x}$ and $4 \mathrm{x}$ ) (Table 2), while the different concentrations of compounds $3 \mathbf{c}$ and $\mathbf{3 g}$ exhibited a very good antifungal activity (2x and $3 \mathrm{x})$ (Table 3 ).

\section{Conclusion}

The synthesized compounds were tested for their antimicrobial activity against three microorganisms and the minimal inhibitory concentrations (MICs) of the tested compounds were determined by the dilution method. The antimicrobial screening showed that many of these newly synthesized compounds have good antimicrobial activities $\mathbf{2 b}, \mathbf{3 c}, \mathbf{3 h}$, and $3 \mathbf{j}$ comparable to streptomycin and fusidic acid as positive standards. On the other hand, when different concentrations of the compound that exhibited a moderate antibacterial activity $\mathbf{3 f}$ were used, this compound exhibit very good antibacterial activity at higher concentrations ( $3 x$ and $4 x$ ) (Table 2), while the different concentrations of compounds $3 \mathrm{c}$ and $\mathbf{3 g}$ exhibited a very good antifungal activity at $2 \mathrm{x}$ and $3 x$ (Table 3).

\section{Acknowledgment}

The authors extend their appreciation to the Deanship of Scientific Research at King Saud University for funding the work through the research group Project no. RGP-VPP-099.

\section{References}

[1] P. Singh, R. L. Goel, and B. P. Singh, "8-acetyl-7-hydroxy-4methyl coumarin as a gravimetric reagent for $\mathrm{Cu}^{2+}$ and $\mathrm{Fe}^{3+}$," Journal of the Indian Chemical Society, vol. 52, pp. 958-959, 1975.

[2] B. F. Perry, A. E. Beezer, R. J. Miles, B. W. Smith, J. Miller, and M. G. Nascimento, "Evaluation of microcalorimetry as a drug bioactivity screening procedure: application to a series of novel Schiff base compounds," Microbios, vol. 45, no. 184-185, pp. 181-191, 1988.

[3] A. Elmali, M. Kabak, and Y. Elerman, "Keto-enol tautomerism, conformations and structure of N-(2-hydroxy5- methylphenyl), 2-hydroxybenzaldehydeimine," Journal of Molecular Structure, vol. 477, no. 1-3, pp. 151-158, 2000.

[4] M. Rahman, M. A. Mridha, and M. A. Ali, "Transition metal complexes of the schiff base derived from S-methyldithiocarbazate with 2-aminobenzaldehyde," Transition Metal Chemistry, vol. 19, no. 2, pp. 237-240, 1994.

[5] M. N. Ibrahim, S. A. I. Sharif, A. N. El-Tajory, and A. A. Elamari, "Synthesis and antibacterial activities of some schiff bases," EJournal of Chemistry, vol. 8, no. 1, pp. 212-216, 2011.

[6] P. Sah, N. Saraswat, and M. Seth, "Synthesis of phthalyl substituted imidazolones and schiff bases as antimicrobial agents," EJournal of Chemistry, vol. 8, no. 1, pp. 427-434, 2011.

[7] M. R. Barbachyn and C. W. Ford, "Oxazolidinone structure-activity relationships leading to linezolid," Angewandte Chemie International Edition, vol. 42, no. 18, pp. 2010-2023, 2003.

[8] A. D. Panchal and P. M. Patel, "Synthesis of N-(5(Substitutedphenyl)-4,5-dihydro-1H-pyrazol-3-yl)-4H-1,2, 4-triazol-4-amine from 4-Amino-4H-1,2,4-triazole," E-Journal of Chemistry, vol. 8, no. 3, pp. 1180-1185, 2011.

[9] A. E. Amr, N. M. Sabry, M. M. Abdalla, and B. F. AbdelWahab, "Synthesis, antiarrhythmic and anticoagulant activities of novel thiazolo derivatives from methyl 2-(thiazol-2ylcarbamoyl)acetate," European Journal of Medicinal Chemistry, vol. 44, no. 2, pp. 725-735, 2009.

[10] A. S. Said, A. E. Amr, N. M. Sabry, and M. M. Abdalla, "Analgesic, anticonvulsant and anti-inflammatory activities of some synthesized benzodiazipine, triazolopyrimidine and bisimide derivatives," European Journal of Medicinal Chemistry, vol. 44, no. 12, pp. 4787-4792, 2009.

[11] S. F. Mohamed, E. M. Flefel, A. E. Amr, and D. N. Abd ElShafy, "Anti-HSV-1 activity and mechanism of action of some new synthesized substituted pyrimidine, thiopyrimidine and thiazolopyrimidine derivatives," European Journal of Medicinal Chemistry, vol. 45, no. 4, pp. 1494-1501, 2010.

[12] M. A. Al-Omar and A. E. Amr, "Synthesis of some new pyridine-2,6-carboxamide-derived schiff bases as potential antimicrobial agents," Molecules, vol. 15, no. 7, pp. 4711-4721, 2010.

[13] R. A. Al-Salahi, M. A. Al-Omar, and A. E. Amr, "Synthesis of chiral macrocyclic or linear pyridine carboxamides from pyridine-2,6-dicarbonyl dichloride as antimicrobial agents," Molecules, vol. 15, no. 9, pp. 6588-6597, 2010. 
[14] A. H. Moustafa, M. G. Assy, A. E. Amr, and R. M. Saber, "Studies on the chemical reactivity of Ethyl 4-sulfanyl-6-methyl-2phenylpyrimidine-5-carboxylate," Current Organic Chemistry, vol. 15, no. 10, pp. 1661-1668, 2011.

[15] S. A. S. Ghozlan, M. A. Al-Omar, A. E. Amr, K. Khalil, and A. A. Abd El-Wahab, "Synthesis and antimicrobial activity of some heterocyclic 2,6-bis(substituted)-1,3,4-thiadiazolo-, oxadiazolo-, and oxathiazolidino-pyridine derivatives from 2,6pyridine dicarboxylic acid dihydrazide," Journal of Heterocyclic Chemistry, vol. 48, no. 5, pp. 1103-1110, 2011.

[16] M. A. Al-Omar, A. E. Amr, and R. A. Al-Salahi, "Anti-inflammatory, analgesic, anticonvulsant and antiparkinsonian activities of some pyridine derivatives using 2,6-disubstituted isonicotinic acid hydrazides," Archiv der Phaemazie, vol. 343, no. 1112, pp. 648-656, 2010.

[17] H. H. Sayed, H. S. Abbas, E. M. H. Morsi, A. E. Amr, and N. A. M. Abdelwahad, "Antimicrobial activity of some synthesized glucopyranosyl-pyrimidine carbonitrile and fused pyrimidine systems," Acta Pharmaceutica, vol. 60, no. 4, pp. 479-491, 2010.

[18] N. A. Abdel-Hafez, A. M. Mohamed, A. E. Amr, and M. M. Abdalla, "Antiarrhythmic activities of some newly synthesized tricyclic and tetracyclic thienopyridine derivatives," Scientia Pharmaceutica, vol. 77, no. 3, pp. 539-553, 2009.

[19] A. E. Amr, M. I. Hegab, A. A. Ibrahiem, and M. M. Abdulla, "Synthesis and reactions of some fused oxazinone, pyrimidinone, thiopyrimidinone, and triazinone derivatives with a thiophene ring as analgesic, anticonvulsant, and antiparkinsonian agents," Monatshefte fur Chemie, vol. 134, no. 10, pp. 1395-1409, 2003.

[20] G. L. Furtada and A. A. Medeiros, "Single-disk diffusion testing (kirby-bauer) of susceptibility of proteus mirabilis to chloramphenicol: significance of the intermediate category," Journal of Clinical Microbiology, vol. 12, no. 4, pp. 550-553, 1980. 

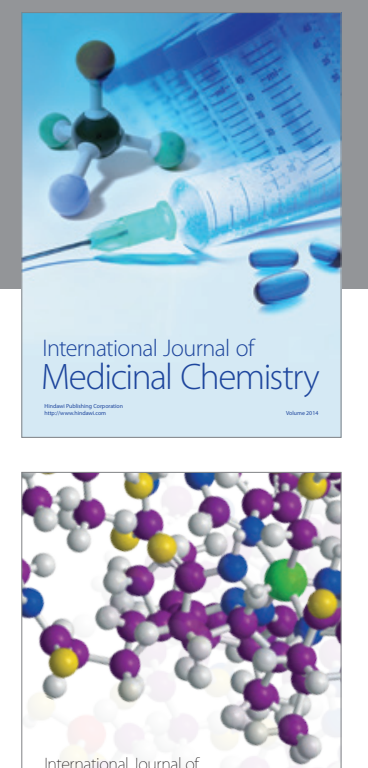

\section{Carbohydrate} Chemistry

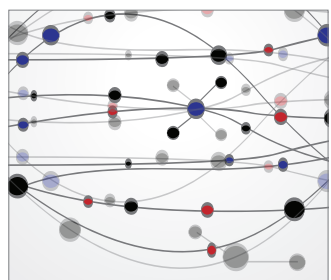

The Scientific World Journal
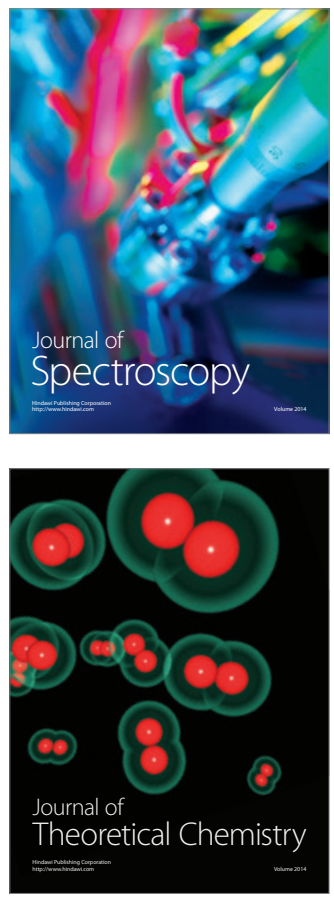
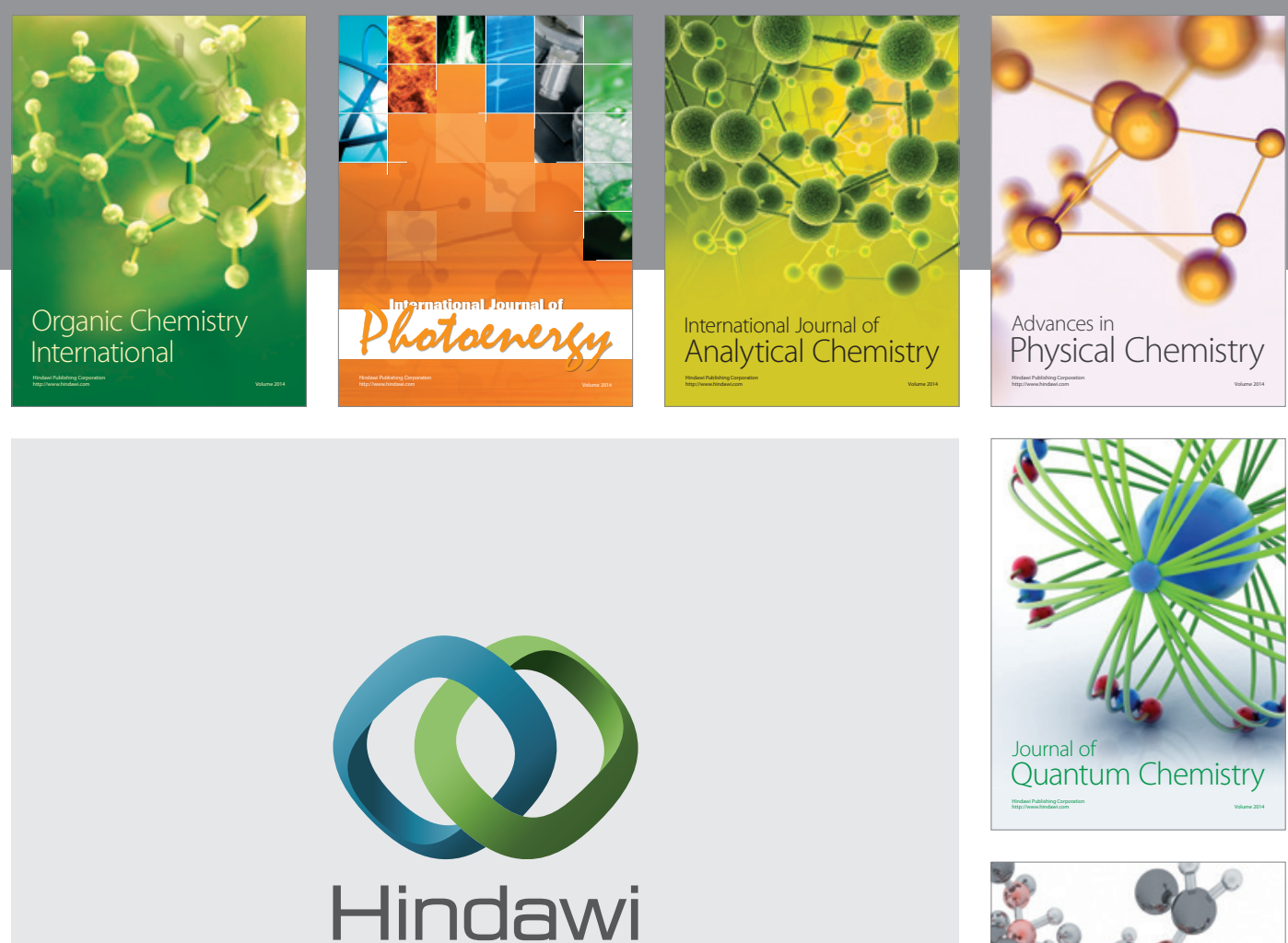

Submit your manuscripts at

http://www.hindawi.com

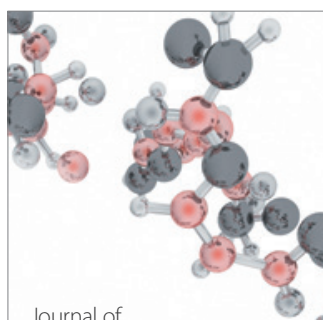

Analytical Methods

in Chemistry

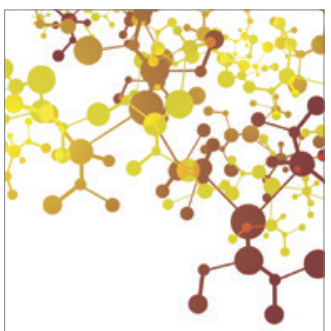

Journal of

Applied Chemistry

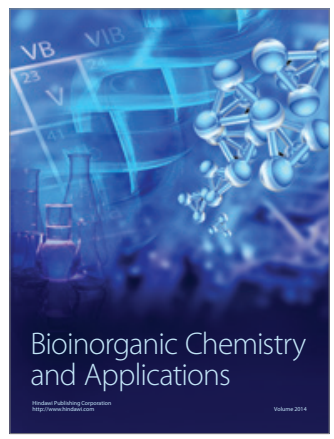

Inorganic Chemistry
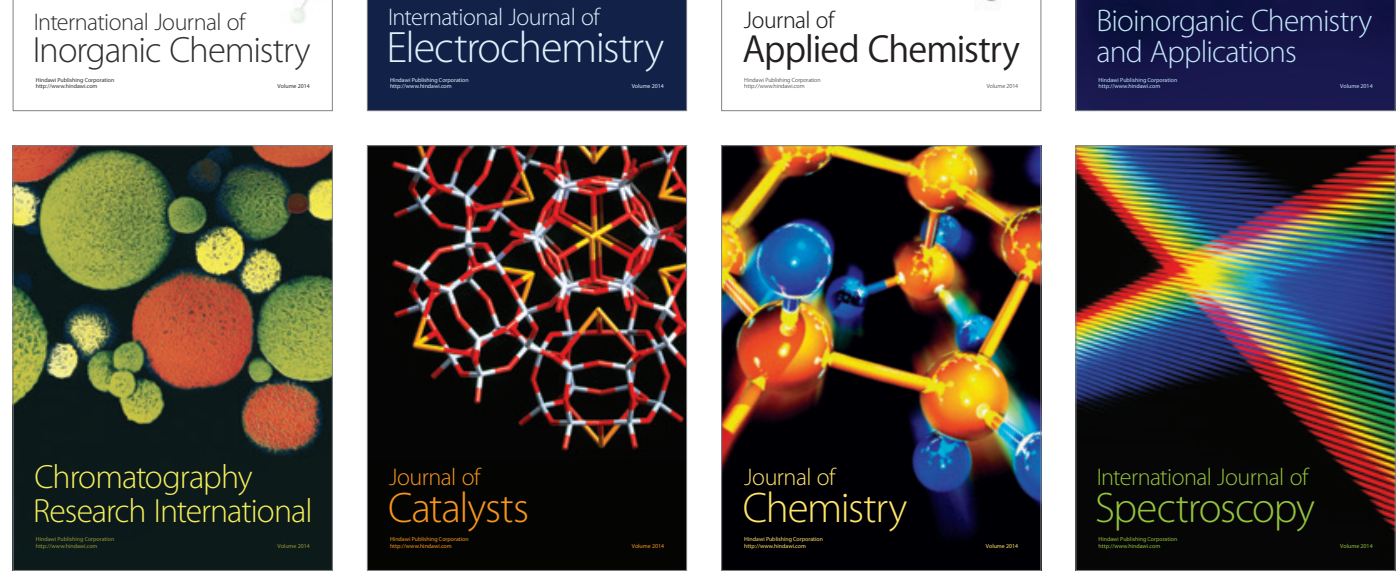